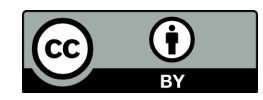

()Auteure. Cette œuvre, disponible à

http://dx.doi.org/10.18162/fp.2020.a192, est distribuée

sous licence Creative Commons Attribution 4.0 International

http://creativecommons.org/licences/by/4.0/deed.fr

\title{
Ethnographie des classes d'accueil au secondaire en milieu montréalais
}

\section{Contexte et problématique de la recherche}

Depuis l'entrée en vigueur de la Charte de la langue française (loi 101) en 1977 au Québec, le système scolaire québécois francophone est devenu un lieu d'accueil pour les immigrants. En effet, les écoles ont dû s'adapter à la diversité linguistique, ethnoculturelle et religieuse (Mc Andrew et Bakhshaei, 2016). Depuis 1998, la Politique d'intégration scolaire et d'éducation interculturelle encadre la mise en œuvre d'une éducation interculturelle et, plus récemment, d'une éducation inclusive (Mc Andrew et Bakhshaei, 2016). Dans cette politique, l'éducation interculturelle se base sur trois principes : une société dont la langue commune est le français; une société démocratique dont la participation est attendue et garantie; et une société pluraliste et ouverte (Ministère de l'Éducation, 1998; Ministère des Communautés culturelles et de l'Immigration, 1990).

Dans la foulée de cette politique, le Québec a mis en place de manière répandue des classes d'accueil pour les élèves ayant besoin de soutien pour apprendre le français ou pour améliorer leurs compétences dans cette langue avant d'être redirigés en classe ordinaire. Cinq modèles d'intégration sont en vigueur: 1) classe d'accueil fermée; 2) classe d'accueil fermée avec aide à l'intégration; 3) intégration partielle; 4) intégration totale dans les classes ordinaires avec soutien à l'apprentissage du français; et 5) intégration totale dans les classes ordinaires sans soutien à l'apprentissage du français (De Koninck et Armand, 2012). 
Jusqu'à présent, les recherches ayant porté sur les classes d'accueil au Québec ont surtout été menées dans le champ de la didactique des langues secondes (De Koninck et Armand, 2012). Cependant, ces recherches ont peu abordé le processus d'ajustement ou, a contrario, de résistance des élèves immigrants dans les classes d'accueil.

\section{Cadre d'analyse de la recherche}

Dans le cas des classes d'accueil au Québec, d'une part, l'école est tenue d'accueillir les différences ethnoculturelles et linguistiques des élèves immigrants en vertu de la Politique d'intégration scolaire et d'éducation interculturelle de 1998. D'autre part, l'école doit appliquer la recommandation de la loi 101, selon laquelle le français est la langue commune de scolarisation. Nous faisons l'hypothèse que cela peut générer des tensions et des conflits entre les élèves et l'équipe-école, car les élèves peuvent réagir à l'autorité et aux règles, notamment linguistiques, imposées par les enseignants de manière directe ou indirecte.

En nous inspirant de Goffman (1961), nous utiliserons d'un côté, dans cette recherche, le concept d'ajustements primaires des élèves des classes d'accueil fermées, c'est-à-dire les ajustements réciproques entre l'individu et l'institution; ce concept inclut l'idée d'une collaboration entre les individus et l'établissement. De l'autre côté, nous mobiliserons le concept d'ajustements secondaires incluant les pratiques adoptées par l'individu pour contester l'ordre établi; il s'agit donc de l'adoption de pratiques dont les moyens ou les fins sont interdits dans l'établissement. L'individu adopte ces stratégies pour réagir aux règles dictées par l'institution. Le concept d'ajustement s'avère directement lié à l'identité des individus (Goffman, 1961).

Nous faisons l'hypothèse qu'il existe à la fois des ajustements primaires et secondaires de la part des jeunes issus de l'immigration vis-à-vis du fonctionnement de la classe d'accueil et des dynamiques à l'œuvre dans l'école en général. Ainsi, dans cette recherche, nous nous pencherons sur les ajustements et réactions aux normes scolaires linguistiques chez des élèves fréquentant les classes d'accueil fermées au secondaire à Montréal, et ce, autant dans des classes d'accueil qu'en dehors des classes d'accueil.

\section{Question et objectifs de la recherche}

Cette recherche vise à répondre à la question suivante : comment les ajustements primaires et secondaires se produisent-ils dans les interactions sociales entre les élèves des classes d'accueil et d'autres acteurs sociaux (enseignants des classes d'accueil, élèves et enseignants des classes ordinaires, direction et autres intervenants) dans l'école?

L'objectif général de cette recherche est de comprendre le processus d'ajustement des élèves issus de l'immigration fréquentant des classes d'accueil. Cet objectif se décline en deux objectifs spécifiques : 1) identifier les ajustements primaires, que ce soit dans la classe d'accueil ou, plus largement, dans l'école; 2) identifier les ajustements secondaires, que ce soit dans la classe d'accueil ou, plus largement, dans l'école. 


\section{Méthodologie de la recherche}

Nous utiliserons l'ethnographie, plus spécifiquement la technique de l'observation participante périphérique, pour analyser les interactions sociales entre les acteurs scolaires d'une école secondaire de Montréal (Pepin, 2011; Vienne, 2005; Whyte, 1993). L'étude ethnographique a pour but de fournir une analyse approfondie avec l'objectif de comprendre le sens que les acteurs sociaux attribuent à leurs interactions sociales (Whyte, 1993).

L'analyse des données tiendra compte de quatre types d'interactions sociales se déroulant en classe d'accueil et ailleurs dans l'école : a) entre les élèves en classe d'accueil; b) entre les élèves et les enseignants en classe d'accueil; c) entre les élèves des classes d'accueil et les élèves des classes ordinaires; d) entre les élèves des classes d'accueil et d'autres acteurs de la communauté éducative présents dans l'institution.

\section{Conclusion}

Dans ce contexte, mener une recherche sur ce sujet est pertinent pour contribuer à améliorer l'intégration des élèves immigrants, qui représentent une forte proportion des élèves du primaire et du secondaire, principalement dans la grande région montréalaise. Il importe de tenter de mieux comprendre leur vécu scolaire jalonné d'interactions sociales quotidiennes.

Notre recherche contribuera à l'avancement des connaissances en analysant, d'un point de vue sociologique et ethnographique, les interactions sociales dans les classes d'accueil au secondaire à Montréal entre les élèves, entre les élèves et les enseignants ainsi qu'entre les élèves des classes d'accueil et les autres membres de la communauté éducative (élèves et enseignants des classes ordinaires, directions d'école et autres intervenants scolaires).

Cette recherche permettra également d'analyser le sentiment d'appartenance ou de non-appartenance de l'élève nouvellement arrivé à son école et, plus largement, au Québec. De plus, des pistes d'intervention destinées aux enseignants de l'accueil et des classes ordinaires ainsi qu'aux directions d'école émaneront de cette recherche, de même que des pistes pour bonifier la formation initiale et continue des maîtres.

\section{Références}

De Koninck, Z. et Armand, F. (2012). Entre métropole et régions, un même raisonnement peut-il soutenir un choix de modèles de services différent pour l'intégration des élèves allophones? Diversité urbaine, 12(1), 69-85.

http://dx.doi.org/10.7202/1019212ar

Goffman, E. (1961). Asylums: essays on the social situation of mental patients and other inmates. New York, NY: Anchor Books.

Mc Andrew, M. et Bakhshaei, M. (2016). La scolarisation des élèves issus de l'immigration et l'éducation interculturelle : historique, situation actuelle et principaux défis. Dans M. Potvin, M.-O. Magnan et J. Larochelle-Audet (dir.), La diversité ethnoculturelle, religieuse et linguistique en éducation : théorie et pratique (p. 19-40). Montréal, QC : Fides Éducation.

Ministère de l'Éducation. (1998). Une école d'avenir : politique d'intégration scolaire et d'éducation interculturelle. Repéré à http://www.education.gouv.qc.ca/fileadmin/site web/documents/education/adaptation-scolaire-services-comp/ $\underline{\text { PolitiqueMatiereIntegrationScolEducInterculturelle UneEcoleAvenir f.pdf }}$ 
Ministère des Communautés culturelles et de l'Immigration. (1990). Au Québec pour bâtir ensemble : énoncé de politique en matière d'immigration et d'intégration. Repéré à http://numerique.banq.qc.ca/patrimoine/details/52327/44435

Pepin, M. (2011). L'ethnographie scolaire : comprendre quoi, comment et pour qui? Recherches qualitatives, (10), 30-46. Repéré à

http://www.recherche-qualitative.qc.ca/documents/files/revue/hors serie/hors serie v10/RQ HS10 Pepin.pdf

Vienne, P. (2005). Mais qui a peur de l'ethnographie scolaire? Éducation et sociétés, (16), 177-192. http://dx.doi.org/10.3917/es.016.0177

Whyte, W. F. (1993). Street corner society: the social structure of an Italian slum (4 éd.). Chicago, IL : University of Chicago Press. http://dx.doi.org/10.7208/chicago/9780226922669.001.0001

\section{Pour citer cet article}

De Oliveira Soares, R. (2020). Ethnographie des classes d'accueil au secondaire en milieu montréalais.

Formation et profession, 28(1), 127-130. http://dx.doi.org/10.18162/fp.2020.a192 